\title{
Efficiency of Femoral Nerve Block for Recovery after Primary Total Knee Arthroplasty
}

\author{
Zeki Tuncel TEKGUL ${ }^{1}$, Mehmet Ugur Bilgin ${ }^{1}$, and Cemil Kayali ${ }^{1}$ \\ ${ }^{1}$ University of Health Sciences Izmir Bozyaka Education and Research Hospital
}

May 3, 2021

\begin{abstract}
Purpose The aim of our study is to evaluate the efficiency of femoral nerve block on recovery after primary total knee arthroplasty. Our primary goal is to provide adequate analgesia with a lower concentration of bupivacaine $(0.125 \%)$ as well as the usual concentration (0.25\%). Comparing the degree of motor block, side effects, postoperative pain scores, opioid consumption, and ambulation and discharge times are our secondary goals. Methods The study was conducted as randomized, controlled and double blind. Sixty three patients were randomised into three groups: G125(n:21) received FNB with 20ml of 0,125\% bupivacaine, G25(n:21) received $10 \mathrm{ml}$ of $0,25 \%$ bupivacaine and GCont(n:21) received no block. FNB was applied with ultrasound guidance after the operation. Pain scores(NRS) at certain time intervals, total opioid consumption, motor block, knee flexion and discharge times were recorded. Results For GCont, pain scores were significantly higher at 2nd, 6th, 12th and 24th hour postoperatively, total opioid consumption was higher(G125:75mg, G25:0mg, GCont:280mg) and first opioid demand time was earlier(G125:12th hour, G25:21st hour GCont:2nd hour). First knee flexion time and mobilization time were also delayed for GCont. G25 had lower scores for quadriceps muscle strength(G25:3/5, G125:4/5, GCont:5/5, Manual Test at 6th Hour), but this did not affect mobilization compared to G125. Conclusion G125 had lower quadriceps muscle strength loss compared to the G25 and earlier mobilization and flexion times, low opioid consumption and low pain scores compared to the control group. In this respect, we believe the femoral nerve block with $0,125 \%$ bupivacaine proves to be a suitable option for analgesia with the potential of maintaining enough movement for recovery after TKA.
\end{abstract}

\section{Efficiency of Femoral Nerve Block for Recovery after Primary Total Knee Arthroplasty}

\section{Abstract \\ Purpose}

The aim of our study is to evaluate the efficiency of femoral nerve block on recovery after primary total knee arthroplasty. Our primary goal is to provide adequate analgesia with a lower concentration of bupivacaine $(0.125 \%)$ as well as the usual concentration $(0.25 \%)$. Comparing the degree of motor block, side effects, postoperative pain scores, opioid consumption, and ambulation and discharge times are our secondary goals.

\section{Methods}

The study was conducted as randomized, controlled and double blind. Sixty three patients were randomised into three groups: G125(n:21) received FNB with $20 \mathrm{ml}$ of 0,125\% bupivacaine, G25(n:21) received 10ml of 0,25\% bupivacaine and GCont(n:21) received no block. FNB was applied with ultrasound guidance after the operation. Pain scores(NRS) at certain time intervals, total opioid consumption, motor block, knee flexion and discharge times were recorded.

\section{Results}


For GCont, pain scores were significantly higher at $2^{\text {nd }}, 6^{\text {th }}, 12^{\text {th }}$ and $24^{\text {th }}$ hour postoperatively, total opioid consumption was higher(G125:75mg, G25:0mg, GCont:280mg) and first opioid demand time was earlier(G125:12 ${ }^{\text {th }}$ hour, G25:21 $1^{\text {st }}$ hour GCont:2 ${ }^{\text {nd }}$ hour). First knee flexion time and mobilization time were also delayed for GCont. G25 had lower scores for quadriceps muscle strength(G25:3/5, G125:4/5, GCont:5/5, Manual Test at $6^{\text {th }}$ Hour), but this did not affect mobilization compared to G125.

\section{Conclusion}

G125 had lower quadriceps muscle strength loss compared to the G25 and earlier mobilization and flexion times, low opioid consumption and low pain scores compared to the control group. In this respect, we believe the femoral nerve block with $0,125 \%$ bupivacaine proves to be a suitable option for analgesia with the potential of maintaining enough movement for recovery after TKA.

Keywords: Total knee arthroplasty; recovery; femoral nerve block; postoperative analgesia

\section{Introduction}

Pain after Total Knee Arthroplasty (TKA) is a challenging condition that should be approached carefully in terms of both patient comfort and an effective rehabilitation process[1-3]. Patients undergo an intensive rehabilitation period after TKA in order to return to a functional life.

Analgesic options for the management of pain after TKA are numerous. Although opioids are effective in relieving pain, they have limited use due to side effects such as nausea, vomiting, constipation, sedation and respiratory depression[4]. Central nerve blocks are capable of providing high-quality analgesia, but the risk of serious complications, dense motor blockage and interference with postoperative anticoagulants limit their use[5,6]. Peripheral nerve blocks may prove to be the most useful analgesic option with minimal side effects, but motor blockage and application difficulties should be considered thoroughly[2].

Determining the perfect use of each method is of vital importance to complete the rehabilitation process without side effects. Femoral nerve block (FNB) is a proven method to reduce opioid consumption and pain scores after TKA, but it also causes loss of quadriceps muscle strength, increase in ambulation time and fall of patients[2]. In this respect, we believe that reducing the motor block effect without modifying the analgesic efficacy of FNB may positively contribute to pain management for TKA patients. For this purpose, we tested whether we could provide effective analgesia without causing motor block by comparing a low concentration of local anesthetics (LA) with a standart concentration which is proven to be effective for analgesia.

The aim of our study is to evaluate the efficiency of femoral nerve block on recovery after primary total knee arthroplasty. Our primary goal is to provide adequate analgesia with a lower concentration of bupivacaine $(0.125 \%)$ as well as the usual concentration $(0.25 \%)$. The secondary aim is to compare motor blockade, side effects, postoperative pain scores, postoperative opioid consumption, ambulation and discharge times among different concentrations of FNB and control groups.

\section{Materials and Methods}

The study was conducted as randomized, controlled and double blind. After the approval of the local ethics committee with decision number: 21032018-02, the patients were enrolled in the study by obtaining their informed consent. A total of sixty three patients were included in the study with the ClinicalTrial record number NCT03623230. Patients older than the age of 18, ASA 1-3 category, who were scheduled to undergo primary unilateral TKA with spinal anesthesia and agreed to participate in the study with no contraindication for spinal or regional anesthesia were included in this study. The criteria for exclusion were history of allergy to LAs, uncontrolled diabetes, peripheral neuropathy, obesity over $35 \mathrm{~kg} / \mathrm{m} 2$, coagulopathy, psychological and emotional lability. Patients who needed additional medication for pain during surgery were also excluded. The enrollment process is further detailed in accordance with the CONSORT 2010 Flow Diagram (Figure 1). 
The study was planned with 3 groups and sixty three patients in total. The control group (GCont) did not receive any intervention and was dressed behind the curtain in the recovery room as if FNB is applied to the patient to provide blindness for both patient and the physician who follows up the patient in the ward. One of the interventional groups (G125) received $20 \mathrm{ml}$ 0.125\% Bupivacaine (Buvasin, 0,5\%, $20 \mathrm{ml}$ single-use vial; VEM Pharmaceuticals, Istanbul) (5 cc 0.5\% Bupivacaine and 15cc Normal Saline (NS)) mixture while

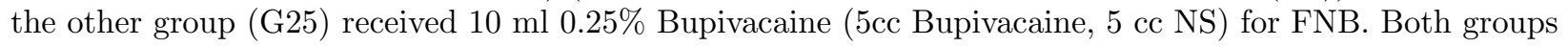
received the same amount of LA with different volume and concentrations. Prior to the operation, patients were randomized by a resident with a simple dice roll. The same resident sorted the patients and tagged the case report forms with patient's numbers only. The randomization information of the patients was passed to the author responsible from statistical analysis after the end of the patient enrollment. Enrollment for each separate group was stopped once the number of patients reached 21.

Patients received spinal anesthesia in the operation room, while seated with legs hanging off the bed, via a 25G pencil-point spinal needle. Then they were taken to the supine position and the operation was allowed if the T6-T9 dermatome blockage was achieved. All patients received $0,02 \mathrm{mg} / \mathrm{kg}$ IV midazolam before and 2lt/min O2 during surgery.

Surgeries were performed by the same surgeon(who is also the co-author) and his team with the same technique. The medial parapatellar approach for incision and cementation was used. In the case report form, patient's demographic and contact information, patient's number(given by the resident in the operating room), operation date, neuraxial block level assessment (evaluated with Pinprick test), length of operation (time from spinal anesthesia to discharge of the patient from the operating room) were recorded during operation. Numeric Rating Scale (NRS, 0-10) scores (at 0., 30., 60. minutes and 2., 6., 12., 24. and 48. hours), reverse of the neuraxial anesthesia induced motor block (Starting to count with the end of surgery, ending when Bromage Score is "0") and FNB induced motor block level (quadriceps strength was evaluated by the Oxford Scale for Manual Muscle Test (MMT) at 6th hour) (Figure 2), nurse controlled opioid administration times and doses, first knee flexion time (voluntarily and pain-free), first $90^{\circ}$ knee flexion time and first time of walking without help were recorded postoperatively.

"NRS", "Bromage Scale" and "MMT" were performed preoperatively(During pre-operative anaesthetic assessment for ASA score) for each patient in order to increase the patient-health worker concordance during data collection as well as to identify abnormal patients. It was questioned whether they could perform knee flexion and $90^{\circ}$ knee flexion(for both knees) or walk on their own. Patients who had issues detected in these tests and interrogations, who had extensive pain scores, contralateral knee or other problems related to ambulation were not included in the study (Figure 1).

Postoperative femoral nerve block was performed in the anesthesia recovery room by an anesthesiologist who is experienced in the femoral nerve block(More than ten years of practice with FNB). While patients were laying in supine position, sterile conditions were provided where artery pulsations were identified in the level of the femoral crease. Femoral artery, vein and nerve were determined with the help of ultrasound (SonoScape S6 Ultrasound; SonoScape Medical Corp, Guangdong, China) and linear ultrasound probe (L741 10.0-5.0 MHz Transducer; SonoScape Medical Corp, Guangdong, China). The needle (Stimuplex A 22Gx2" Insulated Needle; Braun, Melsungen, Germany) was connected to a nerve stimulator (Stimuplex HNS 12; Braun, Melsungen, Germany) which was set up to deliver 0,32 mAh current with $1 \mathrm{~Hz}$ frequency and 0,1 ms length to avoid intraneural injection[7]. The probe was placed in transverse plane and the needle was approached in-plane aspect from lateral to medial. With the order of the performer, half of the solution was injected at 06:00 and the other half at 12:00 direction by the resident who performed randomization. This way, the blindness of the performer was provided for the volume of the local anesthetic solutions. Full circle coverage (Donut Sign) was recognized as a successful block. The solutions that were administered were prepared by the hospital pharmacist for each patient before the procedure. The physician who followed the cases was informed about the successful completion of the procedure.

Standart dosage of intravenous diclofenac $(3 \times 50 \mathrm{mg})$ was administered to all patients in the study for 2 days postoperatively. For the purpose of rescue analgesia, intravenous Tramadol $(1 \mathrm{mg} / \mathrm{kg})$ was ordered with "On 
Demand" footnote and the service nurse was informed about the study and told to administer the medication on patient's request. Patients were periodically visited and were questioned for pain scores (NRS) and motor blockage.

Intraoperative and postoperative follow-ups were performed by health professionals and anesthesiologists who were not associated with the study. Femoral nerve block was performed by the anesthesiologist who carried out the study.

Power analysis was conducted in line with the preliminary study we conducted which included 30 patients in total with the same settings as the main study. Sixth-hour NRS scores(G125: 2.8, G25:2.1 and GCont: 4.7; error variance: 2.1 ) were recorded and $\mathrm{G}^{*}$ Power Version 3.1( $\alpha$-error probability: 0.05 and power: 0.95$)$ was used to determine total sample size. The total sample size was found to be 60 patients but 63 patients were enrolled taking into consideration of a dropout rate of \%5. Statistical Package for Social Sciences (SPSS) Version 18 package program was used for statistical analysis. Parametric test results were presented as mean and standard deviation and non-parametric test results as number and percentage or median and interquartile range (IQR) or total range. The normal distribution of the data was evaluated by the Kolmogorov-Smirnov test. The comparison of quantitative data to normal distribution was performed with one-way ANOVA. The Tukey HSD test was used to determine the difference between groups and the Kruskal Wallis- $\mathrm{H}$ test was used to compare the non-normal distributed quantitative parameters. Mann Whitney U test was used to determine the group which caused the difference. Chi-square test was used to compare the qualitative data. Significance level was determined as $\mathrm{p}<0.05$ for $95 \%$ confidence interval.

\section{Results}

Enrollment of patients occurred between August 10, 2018, and December 12, 2018. 3 eligible patients disagreed to enroll in the study, 11 patients were discarded due to unfitting the including criteria and 4 patients discarded due to unsuccessful FNB (When Donut sign was not identified). (Figure 1)

Demographic, educational, gender, ASA classification data and comparison of groups were presented in Table 1. All groups were homogeneous in these aspects.

Assessment of neuraxial blockade level, length of operation and reverse of the neuraxial anesthesia induced motor block were observed and found to be homogeneous among groups. (Table 2)

Postoperative pain scores (NRS) were recorded at certain time intervals and groups were compared by their pain scores as in Table 3 . GCont had significantly higher pain scores from $2^{\text {nd }}$ to $24^{\text {th }}$ hours postoperatively compared to G125 and G25. No differences between G125 and G25 were demonstrated.

It was recorded whether the patients needed postoperative opioid (intravenous tramadol), and if so, the time and amount of rescue analgesia that were administered was determined. Total opioid use was recorded in milligrams and milligrams $/ \mathrm{kg}$. 52,5\% of the patients who demanded rescue analgesia was in GCont. Total consumption was $280 \mathrm{mg}, 75 \mathrm{mg}$ and $0 \mathrm{mg}$ in GCont, G125, and G25, respectively, as median values. GCont had significantly higher consumption than other groups (p: 0,000) and requested analgesia earlier than the block groups. (at 2nd hour compared to 12th and 21st hour in G125 and G25, respectively, p:0,000) Comparison of the groups in terms of these data was shown in Table 4.

The first knee flexion and the first $90^{\circ}$ knee flexion time of the patients and postoperative hour of ambulation were recorded and compared between the groups and these data were presented in Table 4 . While $90^{\circ}$ knee flexion time was not significantly different, first knee flexion time and ambulation time was significantly longer for GCont. (Table 4)

Results of the manual test for quadriceps muscle and comparison of the groups were presented in Table 4 . Quadriceps muscle strength(MMT) was found to be significantly less in G25. (GCont: 5, G125: 4, G25 3, p:0,000)

Day of discharge and comparison of the groups in terms of this data were shown in Table 4. 


\section{Discussion}

The primary aim of our study was to provide adequate analgesia with different concentrations of bupivacaine $(0,125 \%$ vs. $0,25 \%)$ for the FNB after TKA surgery. As shown in Table 3, pain scores (NRS) in block groups (G125 and G25) were as low as 3 and below 3 in the first 48 hours postoperatively. Compared to the control group, NRS scores of the patients in the block groups were significantly lower at the 2nd, 6th, 12 th and 24th hours. These results are correlated with many studies on this subject[1,2,8-10]. However, the femoral block shows its analgesic properties in minutes, but we detected no difference in NRS scores between the groups in the first two hours after the application[11]. We believe that the spinal anesthesia sensory block is associated with this indifference between the groups. We also detected no difference at the 48th hour. Therefore, we can conclude that the US-guided femoral nerve block with $0.125 \% 20 \mathrm{ml}$ or $0.25 \%$ $10 \mathrm{ml}$ bupivacaine solution provides better analgesia compared to nurse controlled analgesia for at least 24 hours after the application for primary total knee arthroplasty. However, it would be more appropriate to investigate the correct onset and duration of femoral nerve block applied with different LA concentrations and volumes in a patient population that were followed at more precise time intervals and that did not undergo any anesthesia method which could be confused with femoral nerve block efficacy.

In the recovery process after TKA, reducing opioid use has become one of the main objectives and NSAIDs and paracetamol are often included in the analgesia protocols. Peripheral nerve blocks are proven to reduce the opioid need, therefore opioid-related side effects and to increase patient comfort[8]. Our results show that, compared to block groups (G125 and G25), GCont consumed more opioids (75mg and $0 \mathrm{mg}$ to $280 \mathrm{mg}$, p:0,000, median values, respectively) and demanded rescue analgesia earlier (12th and 21st hour to 2nd hour, p:0,000, median values, respectively). Both LA concentrations in our study significantly caused lower opioid consumption compared to the control group. In addition to this, early need for opioid is critical due to the risk of delayed recovery from anesthesia and delayed discharge from post anesthesia care unit (PACU), increased postoperative nausea and vomiting (PONV) and further decreased respiratory drive which is already affected by the sedative medication from earlier. But, since we did not follow any of these side effects, it is not possible to point out any differences regarding these situations. For 48 hours postoperatively, all patients in the control group requested rescue analgesia at least for once. On the other hand, only 8 and 12 patients requested rescue analgesia for G25 and G125 groups, respectively. GCont is found to be statistically different from block groups (p:0,000). In this respect, it is in our view that both concentrations used in FNB reduce opioid consumption for TKA patients, but more studies are needed to compare the opioid-related side effects.

The femoral nerve block is a proven technique to prevent pain after total knee arthroplasty[2-4,9,10], but it may also cause quadriceps muscle weakness which may affect the recovery process[12,13]. Thus, trying to avoid motor block has potential benefits for TKA patients. Using lower concentrations and higher volumes without increasing the dose of bupivacaine for FNB may increase the duration of analgesic action and reduce muscle weakness in the quadriceps muscle. In a prospective experimental study to determine effective concentration for FNB, the minimum effective concentration (EC50) was determined as 0.160\%[14]. But studies such as this one investigate data on anesthetic efficacy of the block for the intraoperative period, not analgesia for the postoperative period. There are studies which used $0.125 \%$ bupivacaine solution for infusion with FNB catheter and it's reported that this concentration does not prevent ambulation[15]. We believe a concentration as low as $0.125 \%$ bupivacaine for single shot FNB shows potential for early ambulation while maintaining adequate analgesia. Our results show that there is no significant difference in the analgesic efficacy and opioid consumption between G25 and G125 group and both drug concentrations provided better outcomes than the control group. In terms of postoperative recovery, patients in the block groups (G25 and G125) were able to move their operated knees earlier (5 and 4.3 hours, respectively: GCont: 8.5 hours, mean values, p:0,020) and ambulated earlier without assistance (20th and 18th hours, respectively, GCont: 24 hours, median values, p:0,001) (Table 4). Some studies show that quadriceps weakness after FNB for TKA can delay ambulation[12,13]. However, many factors may affect ambulation after TKA[16,17]. These can be related to the surgical procedure, patient and anesthesia management. Factors that are associated with anesthesia management may be the anesthesia technique used, the number of invasive procedures and the 
quality of postoperative pain management. In any case, early ambulation after TKA is associated with early discharge time and has been shown to prevent venous thromboembolism[18,19]. However, in order to assess actual causes of delayed ambulation, more comprehensive studies are necessary.

As mentioned earlier, motor block and delayed mobilization are still some of the major concerns when peripheral nerve blocks are the chosen method for postoperative analgesia after TKA. According to the results of the manual test for quadriceps muscle strength, G125 had slightly less scores than the control group and this difference was not statistically significant (Table 4). The same test was found to be significantly lower in the G25 group compared to the control and G125 group (p:0,000). Even though both groups received the same amount of bupivacaine for FNB, G125 experienced less muscle weakness which is statistically significant. We believe this was due to the concentration effect of the LA solution. Despite the difference in muscle strength between the control group and the G125 group, which was statistically not important but clinically important, early ambulation of the G125 group could be interpreted that FNB with 0,125\% bupivacaine may have reduced the muscle strength of the quadriceps, but this effect was not potent enough to prevent ambulation. In fact, it may even facilitate knee flexion and ambulation by relieving pain in the recovery process. On the other hand, femoral block application with $0,25 \%$ bupivacaine caused significant quadriceps muscle weakness compared to other groups. (Table 4)

Adductor Canal Block(ACB) is proven to have less quadriceps muscle strength loss in some studies while providing adequate analgesia which is comparable to FNB after TKA. But FNB is our choice of postoperative analgesia because it's easier to visualize and apply even with an old and low-resolution US device. Another reason is, dressings applied after TKA usually reaches higher than the level of middle or low adductor canal block. Therefore, we aimed to find a solution for the possible negative effects of FNB on quadriceps muscle strength.

One of the limitations of our study originated from the anesthesia method we chose. Confirmation of successful FNB was not convenient as the effect of the spinal anesthesia could continue for hours after surgery. Thus, we had to exclude patients, after the administration of the block, if donut sign for FNB was not identified via US. We believe this is also the reason why the majority of the patients did not report any pain in two hours after the operation.

Another issue is, even though statistical analysis showed no significant difference between the block groups for "first rescue time", this data may actually be clinically important. G125 required intravenous tramadol earlier than G25. This could be due to the number of the patients who demanded tramadol post-operatively was lower than the total sample size(n:12 for G125 and n:8 for G25)

Results showed that discharge times of the control group was significantly longer than other groups. However, the discharge policy of our clinic is not consistent. Discharge decision may vary depending on the weekend or holidays. Socioeconomic status of the patients could also affect this decision. Thus, we believe this data cannot be evaluated properly.

We failed to obtain and compare the data regarding the preoperative analgesic use of the patients. Preoperative use of analgesic medication such as opioid or pregabalin could affect postoperative pain scores and rescue analgesic needs. Although homogeneity in "Control Pain Scores" could mean homogeneity in preoperative analgesic use, we can not interpret this to be correlated accurately.

\section{Conclusions}

Among all three groups, G125 group had lower quadriceps muscle strength loss compared to the G25 group and shortened the ambulation time, earlier flexion times, low opioid consumption and low pain scores compared to the control group. In this respect, we believe that the femoral nerve block with $0,125 \%$ bupivacaine solution proves to be a suitable option for analgesia with the potential of maintaining enough movement for recovery after total knee arthroplasty.

\section{What's already known about this topic?}


-Effective postoperative analgesia in primary total knee arthroplasty ameliorates recovery period.

-Regional nerve blocks may cause motor block which is unfavorable for ambulation in the recovery period.

-Parenteral opioids may augment postoperative complications in elderly patients with comorbidities.

\section{What does this article add?}

-Femoral nerve block is an effective analgesic method in the postoperative period for primer total knee arthroplasty.

$-0.125 \%$ bupivacaine solution provides similar analgesia with decreased quadriceps muscle motor block compared to $0.25 \%$ bupivacaine solution used for single shot femoral nerve block.

\section{References}

1. Karlsen APH, Wetterslev M, Hansen SE, et al.(2017) Postoperative Pain Treatment After Total Hip Arthroplasty: A Systematic Review. PLoS ONE; 12: e0173107

2. Terkawi AS, Mavridis D, Sessler DI, et al.(2017) Pain Management Modalities after Total Knee Arthroplasty. Anesthesiology ; 126: 923-37

3. O'Donnell R, Dolan J. (2018) Anaesthesia and analgesia for knee joint arthroplasty. British Journal of Anaesthesia. BJA Educ ; 18: 8-15

4. Thobhani S, Scalercio L, Elliott CE, et al.(2017) Novel Regional Techniques for Total Knee Arthroplasty Promote Reduced Hospital Length of Stay: An Analysis of 106 Patients. Ochsner J ; 17: 233-8

5. Fowler SJ, Symons J, Sabato S, et al.(2008) Epidural analgesia compared with peripheral nerve blockade after major knee surgery: A systematic review and meta-analysis of randomized trials. $\mathrm{Br} J$ Anaesth ; 100: $154-64$

6. Berninger MT, Friederichs J, Leidinger W, et al.(2018) Effect of local infiltration analgesia, peripheral nerve blocks, general and spinal anesthesia on early functional recovery and pain control in unicompartmental knee arthroplasty. BMC Musculoskelet Disorders ; 2018; 19: 1-9

7. D. O'Flaherty, C.J.L. McCartney, S.C. Ng.(2018) Nerve injury after peripheral nerve blockade - current understanding and guidelines. BJA Education 2018, 18-12, 384-390

8. Paul JE, Arya A, Hurlburt L, et al. (2018)Femoral nerve block improves analgesia outcomes after total knee arthroplasty: a meta-analysis of randomized controlled trials. Anesthesiology ; 113: 1144-62

9. Choi S, O'Hare T, Gollish J, et al.(2016) Optimizing Pain and Rehabilitation after Knee Arthroplasty: A Two-Center, Randomized Trial. Anesth Analg ; 123: 1316-24

10. Chan EY, Fransen M, Parker DA, et al.(2014) Femoral Nerve Blocks For Acute Postoperative Pain After Knee Replacement Surgery (Review). Cochrane Database Syst Rev ; 5: CD009941.

11. Casati A, Fanelli G, Beccaria P, et al.(2000) The effects of the single or multiple injection technique on the onset time of femoral nerve blocks with 0.75\% Ropivacaine. Anesth Analg; 91: 181-4

12. Webb CAJ, Madison S, Goodman SB, et al.(2018) Perioperative Pain Management for Total Knee Arthroplasty: Need More Focus on the Forest and Less on the Trees. Anesthesiology ; 128: 420-1

13. Shah VI, Upadhyay S, Shah K, et al.(2017) Risk of Falling after Femoral Nerve Block for Total Knee Arthroplasty: Periprosthetic Fractures. Journal on Recent Advances in Pain, Sep-Dec; 3 : 125-130

14. Moura ECR, Honda CAO, Bringel RCT, et al.(2016) Minimum effective concentration of bupivacaine in ultrasound-guided femoral nerve block after arthroscopic knee meniscectomy: A randomized, doubleblind, controlled trial. Pain Physician ; 19: E79-86

15. Beebe MJ, Allen R, Anderson MB, et al.(2014) Continuous femoral nerve block using $0.125 \%$ bupivacaine does not prevent early ambulation after total knee arthroplasty. Clin Orthop Relat Res ; 472: $1394-9$

16. Chua MJ, Hart AJ, Mittal R, et al.(2017) Early mobilisation after total hip or knee arthroplasty: A multicentre prospective observational study. PLoS One ; 12: 1-15

17. Ibrahim MS, Alazzawi S, Nizam I, et al.(2013) An evidence-based review of enhanced recovery interventions in knee replacement surgery. Ann R Coll Surg Engl ; 95: 386-9 
18. Pearse EO, Caldwell BF, Lockwood RJ, et al.(2007) Early mobilisation after conventional knee replacement may reduce the risk of postoperative venous thromboembolism. J Bone Joint Surg Br Vol; 89-B: 316-22

19. Hebl JR, Dilger JA, Byer DE, et al.(2008) A Pre-Emptive Multimodal Pathway Featuring Peripheral Nerve Block Improves Perioperative Outcomes After Major Orthopedic Surgery. Reg Anesth Pain Med ; 33: $510-7$

\section{Hosted file}

Figures.pdf available at https://authorea.com/users/377461/articles/520639-efficiency-offemoral-nerve-block-for-recovery-after-primary-total-knee-arthroplasty

\section{Hosted file}

Tables.pdf available at https://authorea.com/users/377461/articles/520639-efficiency-offemoral-nerve-block-for-recovery-after-primary-total-knee-arthroplasty 